\title{
Diphoton signal via Chern-Simons interaction in a warped geometry scenario
}

\author{
Nabarun Chakrabarty* and Biswarup Mukhopadhyaya ${ }^{\dagger}$ \\ Regional Centre for Accelerator-based Particle Physics, \\ Harish-Chandra Research Institute, Chhatnag Road, Jhusi, Allahabad 211019, India \\ Soumitra SenGupta ${ }^{\ddagger}$ \\ Department of Theoretical Physics, Indian Association for the Cultivation of Science, \\ 2A \& $2 B$ Raja S.C. Mullick Road, Kolkata 700032, India
}

\begin{abstract}
The Kalb-Ramond field, identifiable with bulk torsion in a 5-dimensional Randall Sundrum (RS) scenario, has Chern-Simons interactions with gauge bosons, from the requirement of gauge anomaly cancellation. Its lowest Kaluza Klein (KK) mode on the visible 3-brane can be identified with a spin-0 $\mathrm{CP}$-odd field, namely, the axion. By virtue of the warped geometry and Chern-Simons couplings, this axion has unsuppressed interactions with gauge bosons in contrast to ultra-suppressed interactions with fermions. The ensuing dynamics can lead to a peak in the diphoton spectrum, which could be observed at the LHC, subject to the prominence of the signal. Moreover, the results can be numerically justified when the warp factor is precisely in the range required for stabilisation of the electroweak scale.
\end{abstract}

\section{INTRODUCTION}

Suppose there is fundamental spin-0 particle in nature, whose couplings to all Standard Model (SM) particles are extremely suppressed, the only exception being pairs of gauge bosons. In such a case, such interactions should constitute the sum and substance of its phenomenology observable at the Large Hadron Collider (LHC), provided that it is within the kinematic reach of the latter.

Such a situation is not altogether far-fetched. A case in point is a CP-odd spin-0 axion field, in terms of which a Kalb-Ramond (KR) antisymmetric tensor field strength can be defined. As we shall emphasize further in the remaining part of this paper, the KR field exhibits some very interesting properties if it propagates in bulk in a $(1+4)$ dimensional warped geometry scenario as proposed first by Randall and Sundrum. However, the theory is not in general anomaly-free if it arises from a still higherdimensional scenario such as 10-dimensional supergravity. This problem is avoided (see section III) if the KR field is endowed with Chern-Simons (CS) terms couplings with gauge bosons, also propagating in the bulk 1]. On compactification of the warped extra dimension, the CS term has unsuppressed interaction strengths of its zero mode with gauge boson pairs on the $(1+3)$ dimensional visible brane. This unsuppressed character is in turn translated into the interaction of the axion field, in terms of which the zero-mode KR field strength is expressed. On the other hand, the fermionic couplings of the CSaxion turn out to be suppressed as an artefact of the given scenario. With a non-perturbatively acquired mass

\footnotetext{
*nabarunc@hri.res.in

†biswarup@hri.res.in

†tpssg@iacs.res.in
}

of this axion, it can have interesting LHC phenomenology where the CS-driven gluon fusion process can produce it, followed by its decays into gauge boson pairs, of which the most spectacular signal consists in diphoton invariant mass peaks. The expected rates of such peaks are estimated in this paper. We mention in this connection that a recent surge of interest on such diphoton peaks came with the apparent occurrence of a diphoton peak at about $750 \mathrm{GeV}$ in the initial $13 \mathrm{TeV}$ run of the LHC. It was first reported in the preliminary announcement of the $13 \mathrm{TeV}$ run [2, 3] and corroborated in the recent reports in the recent Moriond meeting [4. It led to an avalanche of explanations offered in context of various new physics scenarios(see for a representative list 5 33]). Even though the signal ultimately failed to persist, it could nonetheless glorify the importance of the diphoton final state in detecting a spin- $0 \mathrm{TeV}$-scale resonance, that could indeed be a reality for higher axion masses.

As has been stated already, we present our study in the context of a five-dimensional Randall-Sundrum scenario with bulk space-time torsion identifiable with a KalbRamond tensor field. The massless four-dimensional projection of this field is expressible in terms of an axion which may acquire a mass term non-perturbatively. While this axion field has extremely suppressed coupling with fermions on the four-dimensional visible brane, it can have enhanced interaction with gauge boson pairs via Chern-Simons(CS) terms. Using such CS terms as the driving dynamics, we examine the diphoton production rates in this study. To make matters more practical from the experimenatal perspective, we choose only those values of the warp factor in the five-dimensional geometry, which not only can explain the hierarchy between the Planck and electroweak scales, but also generate KK gauge boson masses above the lower bound set by LHC. We present our results for different axion masses.

This paper is planned as follows. In Section [I] we in- 
troduce the theoretical framework. Prospects of embedding the recently observed resonance in that framework are studied in Section III. A more general discussion on other possible diphoton resonances can be found in Section III. We summarise in Section IV.

\section{THE SCENARIO}

As stated at the beginning, we consider a fivedimensional Randall-Sundrum (RS) scenario, where the extra dimension is a $\mathcal{Z}_{2}$ orbifold of radius $r_{c}$. There are two branes at the orbifold fixed points i.e., $\phi=0$ and $\phi=\pi$, where $\phi$ is the angular variable for the compact co-ordinate. The five-dimensional metric is

$$
d s^{2}=e^{-2 k r_{c}|\phi|} \eta_{\mu \nu} d x^{\mu} d x^{\nu}+r_{c}^{2} d \phi^{2}
$$

with $\eta_{\mu \nu}=\{-,+,+,+\}$ is the Minkowski metric and $k$ (related to the bulk cosmological constant) is of the order of the four-dimensional Planck mass $M_{P}$.

The five-dimensional Planck mass $M$ is related to $M_{P}$ as,

$$
M_{P}^{2}=\frac{M^{3}}{k}\left(1-e^{-2 k r_{c} \pi}\right)
$$

Resolution of the naturalness problem requires $k r_{c} \simeq$ 11.5. Attempts towards finding a warped-geometric explanation to the $750 \mathrm{GeV}$ resonance can be seen in [34-47]

The presence of CS terms can be motivated if the aforesaid scenario is the descendant of, say, a ten dimensional supergravity theory. There the KR field strength tensor is augmented by a set of CS terms in order to cancel gauge anomalies ${ }^{1}$. This anomaly cancellation feature is protected in the 4-dimensional effective theory with a modification of the gauge-KR coupling by an appropriate volume modulus factor.

When the five-dimensional space-time has torsion along with curvature, the torsion field can be identified with the KR field [48 57]. It has been shown earlier that such a field is suppressed in $(1+3)$ dimensions due to the RS geometry, thus explaining why our observed universe is controlled primarily by curvature rather than torsion.

\footnotetext{
1 The massless sector of $\mathrm{D}=10$ supergravity multiplet contains a second rank antisymmetric tensor field $B_{\mu \nu}$ with a corresponding third rank field strength $H_{\mu \nu \alpha}$. This field strength can can also be interpreted as the background space-time torsion. The field $B_{\mu \nu}$ plays a crucial role in canceling the gauge anomaly originating from the one loop hexagon diagrams with six external legs of gauge fields with chiral fermions in the loop. If the third rank field strength is now modified with appropriate Chern-Simons term as $H_{\mu \nu \alpha}=\partial_{[\mu} B_{\nu \alpha]}+\frac{A_{[\mu} F_{\nu \alpha]}}{M_{P}}$, then the corresponding tree diagramm that is generated with Kalb-Ramond field as propagator between the gauge fields at the two vertices exactly cancels the hexagon gauge anomaly [1].
}

The five-dimensional Einstein-Maxwell-Kalb-Ramond (EKMR) action in the Einstein frame reads

$$
\begin{aligned}
S_{e f f}= & \int d^{5} x \sqrt{-G}\left[R-\frac{1}{4} F_{M N} F^{M N}\right. \\
& \left.-\frac{1}{12} \bar{H}_{M N L} \bar{H}^{M N L}\right] \\
\bar{H}_{M N L}= & H_{M N L}+\frac{2}{M^{3 / 2}} B_{[M} F_{N L]}+\frac{2}{M^{3 / 2}} W_{[M}^{i} W_{N L]}^{i} \\
& +\frac{2}{M^{3 / 2}} G_{[M}^{b} G_{N L]}^{b}
\end{aligned}
$$

where a sum over $\mathrm{i}=1,2,3$ and $\mathrm{b}=1,2 . ., 8$ is implied. In addition we have

$$
H_{M N L}=\partial_{[M} B_{N L]}
$$

Here $B_{N L}$ refers to the Kalb-Ramond (KR) two form in five-dimensions. Besides, $B_{M}(x, \phi), W_{M}^{i}(x, \phi)$ and $G_{M}^{b}(x, \phi)$ respectively refer to the $U(1)_{Y}, S U(2)_{L}$ and $S U(3)_{c}$ gauge fields in the bulk with $F_{N L}, W_{N L}^{i}$ and $G_{N L}^{b}$ as the corresponding field strengths ${ }^{2}$. The gauge $S U(2)_{L}$ and $S U(3)_{c}$ gauge indices read $i$ and $b$ respectively. Further, KR gauge invariance allows us to do gauge fixing using $B_{\mu y}=0$.

With the standard model (SM) gauge fields in the bulk, the Kaluza-Klein towers for them as well as the KR field on the visible brane are given by

$$
\begin{aligned}
B_{\mu \nu}(x, \phi) & =\sum_{n=1}^{\infty} B_{\mu \nu}^{n}(x) \frac{\chi^{n}(\phi)}{\sqrt{r_{c}}} \\
C_{\mu}(x, \phi) & =\sum_{n=1}^{\infty} C_{\mu}^{n}(x) \frac{\psi^{n}(\phi)}{\sqrt{r_{c}}}
\end{aligned}
$$

where $C$ stands for the towers corresponding to the SM gauge fields, viz, $B, W$ and $G$. The zero-mode for the KR field obeys the following equation.

$$
\frac{1}{r_{c}^{2}} \frac{d^{2} \chi^{0}}{d \phi^{2}}=0
$$

The solution reads

$$
\chi^{0}(\phi)=c_{1}+c_{2}|\phi|
$$

Continuity of the first derivative of $\chi^{0}(\phi)$ at the orbifold fixed points $\phi=0, \pm \pi$ gives $c_{2}=0 . c_{1}$ is fixed using the orthonormality condition

$$
\int e^{2 k r_{c} \phi} \chi^{m}(\phi) \chi^{n}(\phi) d \phi=\delta_{m n}
$$

\footnotetext{
2 The CS coupling in 5 dimensions will always carry a $\frac{1}{M^{3 / 2}}$ on dimensional grounds. However, the numerical factors may not be strictly same for all gauge fields. For simplicity, we assume universal couplings in this work.
} 
This leads to the following zero-mode profiles.

$$
\begin{aligned}
\chi^{0}(\phi) & =\sqrt{2 k r_{c}} e^{-k r_{c} \pi} \\
\psi^{0}(\phi) & =\frac{1}{\sqrt{2 \pi}}
\end{aligned}
$$

$H_{\mu \nu \lambda}^{0}$, the field strength of $B_{\mu \nu}^{0} H_{\mu \nu \lambda}^{0}$ can be expressed

$$
H_{\mu \nu \lambda}^{0}=\epsilon_{\mu \nu \lambda \rho} \partial^{\rho} a
$$

Here $a$ denotes a CP-odd scalar, called the KR axion. Such an axion acquires a mass term through nonperturbative effects confined to the $\mathrm{TeV}$ brane such as instanton corrections [58. This mass is prima facie a free parameter, and which can be around a $\mathrm{TeV}$ scale $^{3}$. The kinetic terms of $a$ and its coupling to the SM gauge fields via the Chern-Simons terms take the form 52

$$
\begin{aligned}
S_{K i n}= & -\frac{1}{2} \eta^{\mu \nu} \partial_{\mu} a \partial_{\nu} a \\
S_{C S}= & f\left[a B_{\mu \nu} \tilde{B}^{\mu \nu}+a W_{\mu \nu}^{i} \tilde{W}^{i \mu \nu}\right. \\
& \left.+a G_{\mu \nu}^{b} \tilde{G}^{b \mu \nu}\right]
\end{aligned}
$$

where, $f=-\frac{e^{k r_{c} \pi}}{\sqrt{2} \pi k r_{c} M_{P}} \quad$ quantifies the coupling of the axion to the SM gauge bosons. Moreover, $\tilde{B}^{\mu \nu}=\frac{1}{2} \epsilon^{\mu \nu \lambda \rho} B_{\lambda \rho}$ etc. denote the dual of the original field strength.

It should be noted that the CS terms enable the axion to have enhanced coupling to gauge field pairs, by virtue of the specific nature of the warped geometry. In contrast, it has been found [57, 59] that $a$ has interaction to fermion pairs of the form $\sim \frac{e^{-k r_{c} \pi}}{M_{P}}$. As a result, both its production rate via gluon fusion and its diphoton partial decay width are enhanced to an extent to be decided by the acquired mass of the axion on the $\mathrm{TeV}$ brane.

The expressions for the leading order decay widths of $a$ to various $V V$ (pair of gauge-bosons) states are

$$
\begin{aligned}
\Gamma_{a \rightarrow \gamma \gamma} & =\frac{1}{4 \pi} f^{2} m_{a}^{3} \\
\Gamma_{a \rightarrow g g} & =\frac{2}{\pi} f^{2} m_{a}^{3} \\
\Gamma_{a \rightarrow W W} & =\frac{f^{2} m_{a}^{3}}{2 \pi}\left(1-\frac{4 m_{W}^{2}}{m_{a}^{2}}\right)^{3 / 2} \\
\Gamma_{a \rightarrow Z Z} & =\frac{f^{2} m_{a}^{3}}{4 \pi}\left(1-\frac{4 m_{Z}^{2}}{m_{a}^{2}}\right)^{3 / 2}
\end{aligned}
$$

\footnotetext{
${ }^{3}$ It should be noted, however, that the possibility of this mass lying within the reach of the LHC is not predicted by the scenario considered here. The analysis presented by us is more in a 'looking under the lamppost' spirit, since diphoton signals are in any case of great curiosity and phenomenological significance.
}

\section{ANALYSIS STRATEGY AND NUMERICAL PREDICTION.}

We have the following expression for $a$ production cross section via gluon fusion

$$
\sigma_{p p \rightarrow a}(f b)=c_{g g} \frac{\Gamma_{a \rightarrow g g}(G e V)}{m_{a} s} \times 0.3894 \times 10^{12}(\text { III.1 })
$$

Here $c_{g g}$ comes from convoluting over the parton densities.

$$
c_{g g}=\frac{\pi^{2}}{8} \int \frac{d x}{x} g(x) g\left(\frac{m_{a}^{2}}{x s}\right)
$$

For practical purposes, we take $c_{g g} \simeq 2137$. The cross section to the diphoton final state is then straightforwardedly obtained by multiplying with the corresponding branching ratio.

$$
\sigma_{p p \rightarrow a \rightarrow \gamma \gamma}=\sigma_{p p \rightarrow a} \times \frac{\Gamma_{a \rightarrow \gamma \gamma}}{\Gamma_{a}}
$$

The couplings of the axion to the gauge boson pairs are taken to be universal in this study. This makes the branching ratio to a given $V V$ state independent of $k r_{c}$, for a fixed axion mass.

Both the production cross section of $a$ as well as its partial width to $g g$ state are prone to QCD corrections. To encapsulate its effect, one can in principle scale both the production cross section as well the partial width by some $K_{Q C D}$. Considering that the dominant contribution to $\Gamma_{a}$ comes from the $g g$ state and that $K_{Q C D}$ is expected to be greater than unity, its effect in the diphoton cross section largely cancels out.

We mention in this context that we have also implemented the effective Lagrangian into the FeynRules package [66. Subsequently the $p p \rightarrow a$ cross section and its decay rates to various channels were cross checked using the tool MadGraph5_aMC@NLO 67.

The diphoton rate is very sensitive $k r_{c}$, precisely due to its exponential dependence on the latter. So are the masses of the graviton and gauge boson KK excited states. Different values for the parameter $\frac{k}{M_{P l}}$, all less than unity, have been chosen while plotting, so that the bulk curvature is less than the Planck scale 68. Without this constraint, the classical solution of 5-dimensional Einstein's equation cannot be trusted.

With $k=0.7 M_{P l}$ and requiring the first gauge boson KK excitation to be heavier than $3.4 \mathrm{TeV}$ [69] ${ }^{4}$ leads

\footnotetext{
4 The limit from the non-observation of dilepton peaks, as obtained in [69, depends on the decay width of the heavier vector boson. The limit is as strong as $4.05 \mathrm{TeV}$ for the spin- 1 particle having SM-like couplings to to fermions, while it could be $3 \mathrm{TeV}$ or lesser with narrower widths. Keeping in mind the fact that a first excited spin-1 KK state has weaker coupling than in the $\mathrm{SM}$, we have taken the limit, somewhat conservatively, as 3.4
} 
to $k r_{c} \leq 11.72$. For a given $k r_{c}$, this upper bound gets tighter upon using a smaller value for $k$.

The initial results of the $13 \mathrm{TeV}$ collisions have practically ruled out a diphoton resonance of mass less than $750 \mathrm{GeV}$ that has 'reasonable' interaction strength with SM particles. For the spin-zero axion considered here, an exception may occur only if the warp factor $k r_{c}$ is way below what is required for addressing the hierarchy between the Planck and electroweak scales. However, higher masses are still within reach. We display the diphoton rates for axion masses around $1 \mathrm{TeV}, 1.5 \mathrm{TeV}$ and $2.5 \mathrm{TeV}$ in Fig. 1. As expected, the rate goes down as the axion gets heavier. It is seen that a $1 \mathrm{TeV}$ axion can have a production cross section of $\simeq 5 \mathrm{fb}$ at $\sqrt{s}=13 \mathrm{TeV}$, for $k r_{c}=11.6$. This goes up to $\simeq 10 \mathrm{fb}$ for $k r_{c}=11.7$. Dynamically enhancing the rate by increasing $k r_{c}$ to still higher values will invariably come into conflict with the requirement of heavy KK states. Hence, to get an appreciable significance, one must wait till the LHC-13 gathers more data. The sensitivity however is marginally better for $\sqrt{s}=14 \mathrm{TeV}$. For example, a $1 \mathrm{TeV}$ axion can yield $\mathrm{a} \simeq 18 \mathrm{fb}$ cross section for the diphotons in this case. In principle, the other decay modes (such as digluons) can also be observed at the LHC. However, the observability of these are perhaps more challenging than that for the diphotons, since rates of ZZ - peaks undergo branching fraction suppression, while dijet peaks from gluon pairs are swamped by the background.

Fig. 2 quantitatively depicts the reach of the $13 \mathrm{TeV}$ LHC in detecting a diphoton resonance that has its origin in the framework under study. While a $1 \mathrm{fb}$ diphoton cross section can be predicted even for a $1.9 \mathrm{TeV}$ axion, a more sizeable rate of $10 \mathrm{fb}$ demands a much lighter axion $(\leq 1 \mathrm{TeV})$. The collider must gather appreciable luminosity to discern a feeble resonant diphoton rate $(<$ $1 \mathrm{fb}$, say) from the background. If, upon accumulation of the requisite luminosity, one notices such 'clean' diphoton peaks, the next step would be to see if the $W W$ and $Z Z$ peaks with correlated strengths are also noticeable. In case they are, one has to look further for the presence or absence of corresponding peaks with fermions. If such peaks are absent, then one will be directed to spinless particles which have unsuppressed couplings with gauge boson pairs but no interactions with fermions. One possible interpretation of such coincidence of observed phenomena may be CS dynamics embedded in a warped geometry. The exact estimate of the LHC reach for higher masses will require a careful analysis of cuts and their efficiencies corresponding to the high diphoton invariant mass.

$\mathrm{TeV}$. Nonetheless, this leads to a stronger upper limit on $k r_{c}$, than what can be imposed from KK graviton searches, given the lower bound on the mass of the first graviton KK excited stands at $2.68 \mathrm{TeV}$ [0].
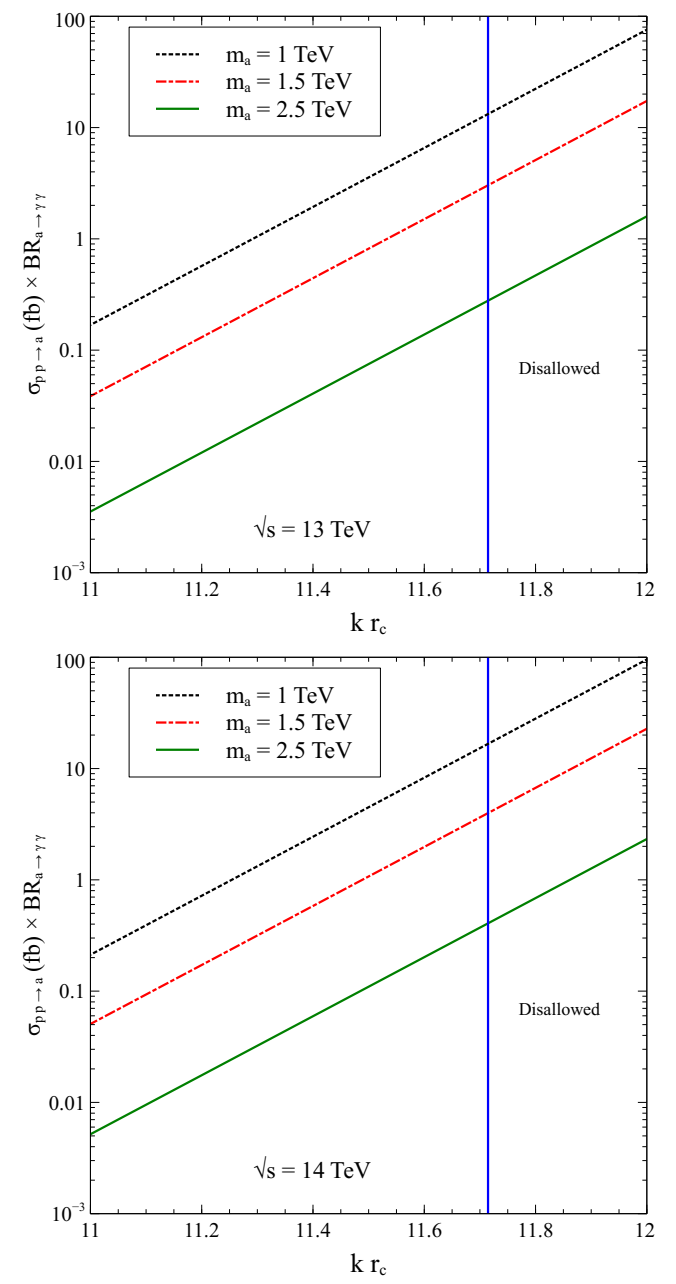

FIG. 1: Diphoton cross sections at LHC-13 and LHC-14 for $m_{a}=1,1.5$ and $2.5 \mathrm{TeV}$. The region on the right of the vertical line corresponds to a KK gauge boson below the existing bound.

\section{SUMMARY}

In conclusion, a bulk KR field in a 5-dimensional bulk with RS warped geometry can be connected with an axion in 4-dimensions, which, with a non-perturbatively acquired mass, can lead to a bump in the diphoton spectrum. The most notable feature of this framework is that the production as well as decay of the axion is triggered by 5 -dimensional Chern-Simons terms which are not ad hoc introduction but necessitated by the cancellation of gauge anomalies. Even if one makes the simplifying assumption of universal CS couplings for all gauge bosons, the diphoton rate for a $1 \mathrm{TeV}$ axion can attain sizeable values at the $13 \mathrm{TeV}$ LHC. However, a more realistic estimate is only expected to emerge after detector simulation is carried out. We remind that the novelty of the suggested scenario lies in the fact that the very same the warp factor, which is responsible for the reported dipho- 


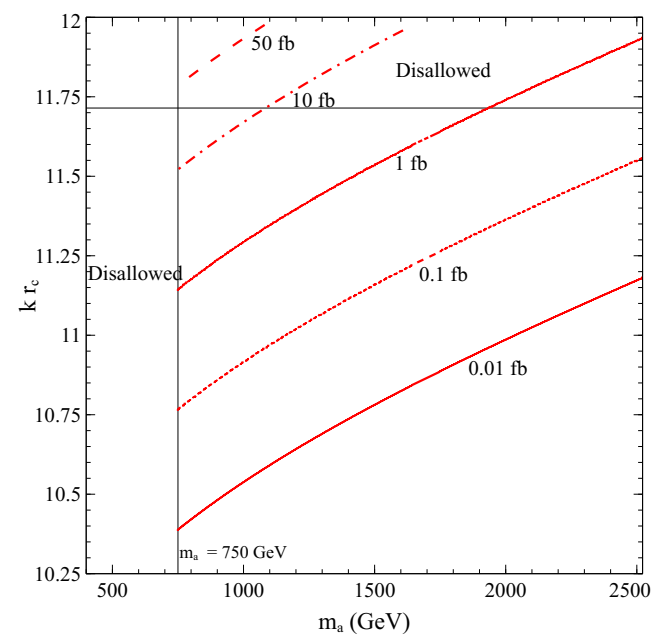

FIG. 2: Contours of constant cross section in the $m_{a}$ vs $k r_{c}$ plane. The region to the left of the vertical line is disallowed from the non-observation of a diphoton resonance below 750 $\mathrm{GeV}$. Similarly, the region above the horizontal line is disallowed from KK gauge boson searches. ton rate, can bridge the hierarchy between the Planck and electroweak scales. The visibility of a higher diphoton peak can in principle improve in the upcoming 14 $\mathrm{TeV}$ runs. In all, the final word on a CP-odd scalar around the $\mathrm{TeV}$ scale, interacting with the gauge bosons through CS terms, will certainly emerge from accumulating more experimental data. Nonetheless, we find the above correlation rather thought-provoking.

\section{Acknowledgement}

This work of NC and BM was partially supported by funding available from the Department of Atomic Energy, Government of India for the Regional Centre for Accelerator-based Particle Physics [RECAPP], HarishChandra Research Institute. SSG acknowledges the hospitality of RECAPP while this work was in progress. We also thank Shankha Banerjee, Subhadeep Mondal and Ashoke Sen for helpful discussions.
[1] M. B. Green and J. H. Schwarz, Physics Let-

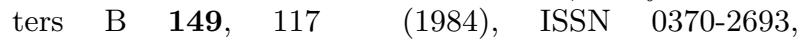
http://www.sciencedirect.com/science/article/ pii/037026938491565X

[2] T. A. collaboration(2015)

[3] C. Collaboration(2015)

[4] T. A. collaboration(2016)

[5] F. Staub et al.(2016), arXiv:1602.05581 [hep-ph]

[6] X.-F. Han and L. Wang, Phys. Rev. D 93, 055027 (Mar 2016), http://link.aps.org/doi/10.1103/PhysRevD. 93.055027

[7] Y. Nakai, R. Sato, and K. Tobioka(2015), arXiv:1512.04924 [hep-ph]

[8] G. Arcadi, P. Ghosh, Y. Mambrini, and M. Pierre(2016), arXiv:1603.05601 [hep-ph]

[9] P. S. B. Dev, R. N. Mohapatra, and Y. Zhang, Journal of High Energy Physics 2016, 1 (2016), ISSN 1029-8479, http://dx.doi.org/10.1007/JHEP02(2016) 186

[10] D. Bardhan, P. Byakti, D. Ghosh, and T. Sharma(2016), arXiv:1603.05251 [hep-ph]

[11] M. Perelstein and Y.-D. Tsai(2016), arXiv:1603.04488 [hep-ph]

[12] J. Bernon, A. Goudelis, S. Kraml, K. Mawatari, and D. Sengupta(2016), arXiv:1603.03421 [hep-ph]

[13] C.-W. Chiang, H. Fukuda, M. Ibe, and T. T. Yanagida(2016), arXiv:1602.07909 [hep-ph]

[14] U. Ellwanger and C. Hugonie(2016), arXiv:1602.03344 [hep-ph]

[15] C. Han, T. T. Yanagida, and N. Yokozaki, Phys. Rev. D 93, 055025 (Mar 2016), http://link.aps.org/doi/10. 1103/PhysRevD.93.055025

[16] J. Chakrabortty, A. Choudhury, P. Ghosh, S. Mondal, and T. Srivastava(2015), arXiv:1512.05767 [hep-ph]

[17] A. Falkowski, O. Slone, and T. Volansky, Journal of High Energy Physics 2016, 1 (2016), ISSN 1029-8479, http:
//dx.doi.org/10.1007/JHEP02(2016) 152

[18] R. Benbrik, C.-H. Chen, and T. Nomura, Phys. Rev. D 93, 055034 (Mar 2016), http://link.aps.org/doi/10. 1103/PhysRevD.93.055034

[19] D. Curtin and C. B. Verhaaren, Phys. Rev. D 93, 055011 (Mar 2016), http://link.aps.org/doi/10. 1103/PhysRevD.93.055011

[20] A. Angelescu, A. Djouadi, and G. Moreau, Physics

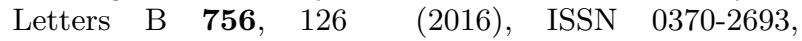
http://www.sciencedirect.com/science/article/ pii/S037026931600160X

[21] E. Gabrielli, K. Kannike, B. Mele, M. Raidal, C. Spethmann, and H. Veerme, Physics Letters B 756, 36 (2016), ISSN 0370-2693, http://www.sciencedirect. com/science/article/pii/S0370269316001659

[22] K. Harigaya and Y. Nomura, Physics Letters B 754, 151 (2016), ISSN 0370-2693, http://www.sciencedirect. com/science/article/pii/S0370269316000381

[23] Y. Mambrini, G. Arcadi, and A. Djouadi, Physics

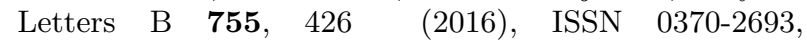
http://www.sciencedirect.com/science/article/ pii/S0370269316001453

[24] D. Buttazzo, A. Greljo, and D. Marzocca, The European Physical Journal C 76, 1 (2016), ISSN 1434-6052, http: //dx.doi.org/10.1140/epjc/s10052-016-3970-7

[25] J. J. Heckman, Nuclear Physics B 906, 231 (2016), ISSN 0550-3213, http://www.sciencedirect.com/science/ article/pii/S0550321316000754

[26] H. Davoudiasl and C. Zhang, Phys. Rev. D 93, 055006 (Mar 2016), http://link.aps.org/doi/10. 1103/PhysRevD.93.055006

[27] A. Salvio and A. Mazumdar, Physics Letters B 755, 469 (2016), ISSN 0370-2693, http://www.sciencedirect. com/science/article/pii/S0370269316001532

[28] L. A. Anchordoqui, I. Antoniadis, H. Goldberg, 
X. Huang, D. Lst, and T. R. Taylor, Physics Letters B 755, 312 (2016), ISSN 0370-2693, http://www.sciencedirect.com/science/article/ pii/S0370269316001209

[29] T. Nomura and H. Okada, Physics Letters B 755, 306 (2016), ISSN 0370-2693, http://www.sciencedirect. com/science/article/pii/S0370269316001180

[30] U. Aydemir and T. Mandal(2016), arXiv:1601.06761 [hep-ph]

[31] S. F. King and R. Nevzorov, Journal of High Energy Physics 2016, 1 (2016), ISSN 1029-8479, http://dx. doi.org/10.1007/JHEP03(2016) 139

[32] J. S. Kim, J. Reuter, K. Rolbiecki, and R. R. de Austri, Physics Letters B 755, 403 (2016), ISSN 0370-2693, http://www.sciencedirect.com/science/article/ pii/S0370269316001374

[33] C. Csáki, J. Hubisz, and J. Terning, Phys. Rev. D 93, 035002 (Feb 2016), http://link.aps.org/doi/10. 1103/PhysRevD.93.035002

[34] A. Carmona(2016), arXiv:1603.08913 [hep-ph]

[35] J. L. Hewett and T. G. Rizzo(2016), arXiv:1603.08250 [hep-ph]

[36] C. Csaki and L. Randall(2016), arXiv:1603.07303 [hep$\mathrm{ph}]$

[37] A. Falkowski and J. F. Kamenik(2016), arXiv:1603.06980 [hep-ph]

[38] M. Bauer, C. Hoerner, and M. Neubert(2016), arXiv:1603.05978 [hep-ph]

[39] V. Sanz(2016), arXiv:1603.05574 [hep-ph]

[40] S. B. Giddings and H. Zhang(2016), arXiv:1602.02793 [hep-ph]

[41] A. Martini, K. Mawatari, and D. Sengupta(2016), arXiv:1601.05729 [hep-ph]

[42] C. Cai, Z.-H. Yu, and H.-H. Zhang(2015), arXiv:1512.08440 [hep-ph]

[43] A. Ahmed, B. M. Dillon, B. Grzadkowski, J. F. Gunion, and Y. Jiang(2015), arXiv:1512.05771 [hep-ph]

[44] M. T. Arun and P. Saha(2015), arXiv:1512.06335 [hep$\mathrm{ph}]$

[45] P. Cox, A. D. Medina, T. S. Ray, and A. Spray(2015), arXiv:1512.05618 [hep-ph]

[46] B. M. Dillon and V. Sanz(2016), arXiv:1603.09550 [hep$\mathrm{ph}]$

[47] D. Bardhan, D. Bhatia, A. Chakraborty, U. Maitra, S. Raychaudhuri, and T. Samui(2015), arXiv:1512.06674 [hep-ph]

[48] M. Kalb and P. Ramond, Phys. Rev. D 9, 2273 (Apr 1974), http://link.aps.org/doi/10.1103/PhysRevD. 9.2273

[49] S. Kar, P. Majumdar, S. SenGupta, and S. Sur, Classical and Quantum Gravity 19, 677 (2002), http://stacks. iop.org/0264-9381/19/i=4/a=304

[50] P. Majumdar and S. SenGupta, Classical and Quantum Gravity 16, L89 (1999), http://stacks.iop.org/ $0264-9381 / 16 / i=12 / a=102$

[51] B. Mukhopadhyaya, S. Sen, S. Sen, and S. SenGupta,
Phys. Rev. D 70, 066009 (Sep 2004), http://link.aps . org/doi/10.1103/PhysRevD.70.066009

[52] D. Maity, S. SenGupta, and S. Sur, Phys. Rev. D 72, 066012 (Sep 2005), http://link.aps.org/doi/10. 1103/PhysRevD.72.066012

[53] D. Maity, S. Roy, and S. SenGupta, Phys. Rev. D 77, 015010 (Jan 2008), http://link.aps.org/doi/10. 1103/PhysRevD.77.015010

[54] B. Mukhopadhyaya and S. Sengupta, Physics Letters B 458, 8 (1999), ISSN 0370-2693, http://www.sciencedirect.com/science/article/ pii/S037026939900605X

[55] B. Mukhopadhyaya, S. Sengupta, and S. Sur, Modern Physics Letters A 17, 43 (2002)

[56] D. Maity and S. SenGupta, Classical and Quantum Gravity 21, 3379 (2004), http://stacks.iop.org/ $0264-9381 / 21 / i=14 / a=004$

[57] B. Mukhopadhyaya, S. Sen, and S. SenGupta, Phys. Rev. Lett. 89, 121101 (Aug 2002), http://link.aps. org/doi/10.1103/PhysRevLett.89.121101

[58] Y. Nomura, T. Watari, and T. Yanagida, Phys. Rev. D 61, 105007 (Apr 2000), http://link.aps.org/doi/10. 1103/PhysRevD.61.105007

[59] A. Das, B. Mukhopadhyaya, and S. SenGupta, Phys. Rev. D 90, 107901 (Nov 2014), http://link.aps.org/ doi/10.1103/PhysRevD.90.107901

[60] L. Aparicio, A. Azatov, E. Hardy, and A. Romanino(2016), arXiv:1602.00949 [hep-ph]

[61] N. D. Barrie, A. Kobakhidze, M. Talia, and L. Wu, Phys. Lett. B755, 343 (2016), arXiv:1602.00475 [hep-ph]

[62] A. Pilaftsis, Phys. Rev. D 93, 015017 (Jan 2016), http: //link.aps.org/doi/10.1103/PhysRevD.93.015017

[63] E. Molinaro, F. Sannino, and N. Vignaroli(2015), arXiv:1512.05334 [hep-ph]

[64] T. Higaki, K. S. Jeong, N. Kitajima, and F. Takahashi, Physics Letters B 755, 13 (2016), ISSN 0370-2693, http://www.sciencedirect.com/science/article/ pii/S0370269316000745

[65] I. Ben-Dayan and R. Brustein(2016), arXiv:1601.07564 [hep-ph]

[66] A. Alloul, N. D. Christensen, C. Degrande, C. Duhr, and B. Fuks, Computer Physics Communications 185, 2250 (2014), ISSN 0010-4655, http://www.sciencedirect. com/science/article/pii/S0010465514001350

[67] J. Alwall, R. Frederix, S. Frixione, V. Hirschi, F. Maltoni, O. Mattelaer, H. S. Shao, T. Stelzer, P. Torrielli, and M. Zaro, JHEP 07, 079 (2014), arXiv:1405.0301 [hep-ph]

[68] H. Davoudiasl, J. L. Hewett, and T. G. Rizzo, Phys. Rev. Lett. 84, 2080 (2000), arXiv:hep-ph/9909255 [hep-ph]

[69] Search for new high-mass resonances in the dilepton final state using proton-proton collisions at $\sqrt{s}=13 \mathrm{TeV}$ with the ATLAS detector, Tech. Rep. ATLAS-CONF2016-045 (CERN, Geneva, 2016) http://cds.cern.ch/ record/2206127

[70] T. A. collaboration 